Archive for

Organic Chemistry

Arkivoc 2017, part iii, 316-325

\title{
A convenient modified synthesis of 5-pyridinyl-1,3,4-thiadiazole-2-carboxamides
}

K. A. Myannik, ${ }^{a}$ V. N. Yarovenko, ${ }^{a *}$ G. M. Rodionova, ${ }^{b}$ T. K. Baryshnikova, ${ }^{a}$ and M. M. Krayushkin ${ }^{a *}$

${ }^{a}$ N.D. Zelinsky Institute of Organic Chemistry, Russian Academy of Sciences, Moscow 119991, Russia

${ }^{b}$ I.M. Sechenov First Moscow State Medical University, Moscow 119991, Russia

E-mail: yarov@ioc.ac.ru mkray@ioc.ac.ru

Dedicated to Prof. Oleg Rakitin on the occasion of his $65^{\text {th }}$ birthday

Received 06-23-2017

Accepted 08-11-2017

Published on line $08-31-2017$

\section{Abstract}

A general one-pot procedure is developed for the synthesis of 5-pyridinyl-1,3,4-thiadiazole-2-carboxamides by the reaction of pyridine carboxaldehydes with oxamic acid thiohydrazides.

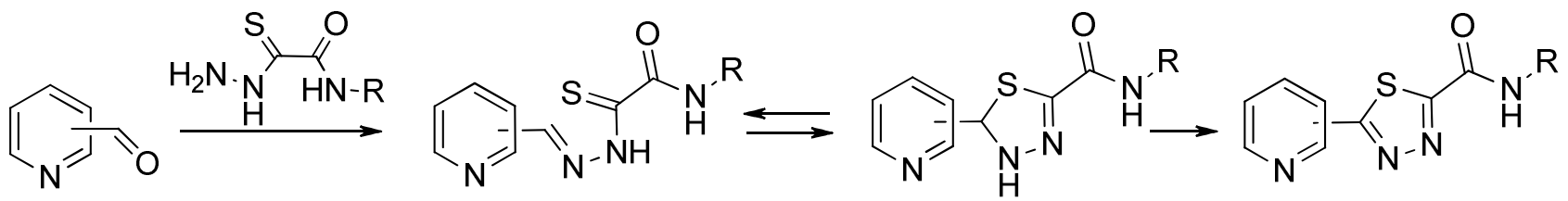

Keywords: Pyridine carboxaldehydes, 1,3,4-thiadiazoles, oxamic acid thiohydrazides, pyridinyl thiadiazoles, carboxamides 


\section{Introduction}

1,3,4-Thiadiazoles possess a broad spectrum of biological activities. ${ }^{1-4}$ Pyridinyl-1,3,4-thiadiazolecarboxamides, in particular, are of great interest. The latter compounds have attracted attention as insecticides, ${ }^{5}$ inhibitors of the type III secretion system of pathogenic bacteria, ${ }^{6}$ and cysteine protease inhibitors. ${ }^{7}$ This encouraged us to develop a facile synthesis of 5-pyridin-2,3 or 4-yl-1,3,4-thiadiazole-2-carboxamides.

The main multistep method for the synthesis of 5-pyridinyl-1,3,4-thiadiazolecarboxamides involves the acylation of pyridine carbohydrazides with ethyl chlorooxoacetate, the cyclization to the thiadiazole ring, the saponification of the ester group to the carboxyl one, the activation of the latter and the reaction with amines $^{5,7}$ (as described for the pyridin-4-yl isomer in Scheme 1). However, the synthesis of 5-pyridin-2-yl-1,3,4thiadiazolecarboxamides was not described by this method.<smiles>CCOC(=O)OC[C@@H]1C[C@@H](C)OCCO1</smiles>

1

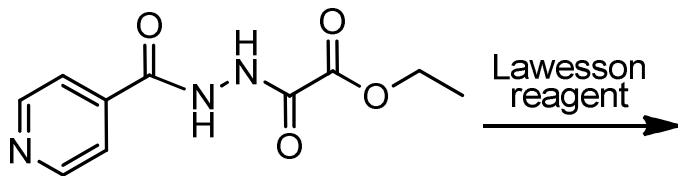

2<smiles>O=C(Nc1ccccc1)Nc1nnc(-c2ccncc2)s1</smiles>

5<smiles>CCOC(=O)c1nnc(-c2ccncc2)s1</smiles>

3<smiles>C[13CH]=O</smiles>

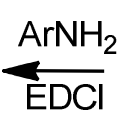

$\overline{\mathrm{EDCl}}$<smiles>O=C(O)c1nnc(-c2ccncc2)s1</smiles>

4

Scheme 1. Synthesis of 5-pyridin-4-yl-1,3,4-thiadiazolecarboxamides.

In this work, we report a new general procedure for the synthesis of 1,3,4-thiadiazole-2-carboxamides substituted at the 5-position by a pyridin-2,3, or 4-yl group.

\section{Results and Discussion}

One of the known methods reported for the synthesis of 1,3,4-thiadiazoles is based on the oxidation of thiohydrazones produced by the reaction of thiohydrazides with aldehydes. ${ }^{8}$

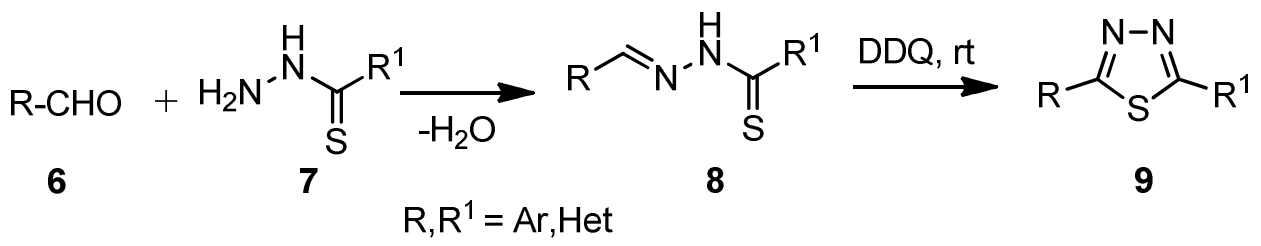

Scheme 2. Synthesis of thiadiazoles from thiohydrazones. 
We have extended this approach to the synthesis of 1,3,4-thiadiazole-2-carboxamides containing a pyridin-2, 3, or 4-yl group at 5-position based on the previously unknown pyridine-containing hydrazones of oxamic acid thiohydrazides $14 \mathrm{a}-\mathrm{m}$. The latter compounds $14 \mathrm{a}-\mathrm{m}$ were synthesized according to Scheme 3 via the reaction of available $\alpha$-chloroacetamides $12 \mathrm{a}-\mathrm{m}$ with a previously prepared solution of elemental sulfur and morpholine followed by the addition of hydrazine-hydrate. ${ }^{9}$<smiles>[R]NC(=O)C(=O)NN</smiles>

$$
\begin{aligned}
& \mathrm{R}=3,5(\mathbf{a}) / 2,4(\mathbf{b}) / 2,5(\mathbf{c}) / 3,4(\mathbf{d}) / 2,6(\mathbf{e})-(\mathrm{MeO})_{2}-\mathrm{C}_{6} \mathrm{H}_{3} \text {, } \\
& \text { 2,4- } \mathrm{Cl}_{2}-\mathrm{C}_{6} \mathrm{H}_{3} \text { (f), 2-Py(g), } \mathrm{Ph}(\mathbf{h}), 4-\mathrm{Br}-\mathrm{C}_{6} \mathrm{H}_{4}(\mathbf{i}), 3-\mathrm{Cl}-\mathrm{C}_{6} \mathrm{H}_{4}(\mathbf{j}) \text {, } \\
& \text { 3- } \mathrm{MeO}-\mathrm{C}_{6} \mathrm{H}_{4}(\mathbf{k}), 2,6-\left(\mathrm{CH}_{3}\right)_{2}-\mathrm{C}_{6} \mathrm{H}_{4}(\mathrm{I}), 2-\mathrm{CH}_{3}-\mathrm{C}_{6} \mathrm{H}_{4}(\mathbf{m})
\end{aligned}
$$

Scheme 3. Synthesis of oxamic acid thiohydrazides.

We investigated the effect of solvents, catalysts, and temperature on the yield of thiadiazole $18 \mathrm{~g}$ produced by the reaction of 4-pyridine carboxaldehyde $15 \mathrm{c}$ with availible oxamic acid $\mathrm{N}$-phenyl-2thiohydrazide $\mathbf{1 4 h}$. It was found that the reaction performed in different solvents (Table 1 ) does not stop at the formation of hydrazone $\mathbf{1 6 g}$ or the equilibrium tautomer form dihydrothiadiazole $\mathbf{1 7 g}$, but immediately gives thiadiazole $\mathbf{1 8 g}$ after auto-oxidation step with air oxygen (Scheme 4). In inert atmosphere the performed reaction does not lead to unoxidized compound (hydrazone $\mathbf{1 6 g}$ or dihydrothiadiazole $\mathbf{1 7 g}$ ).

Table 1. Effect of solvents on the yield of thiadiazole $\mathbf{1 8 g}$

\begin{tabular}{cc}
\hline Solvent & Yield \\
\hline DMF & - \\
MeCN & $60 \%$ \\
THF & $30 \%$ \\
MeOH & $80 \%$ \\
EtOH & $80 \%$ \\
\hline
\end{tabular}<smiles>O=Cc1cccnc1</smiles>

15 a: 2-Py

b: $3-P y$ c: 4-Py

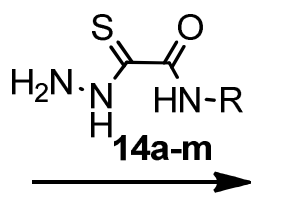<smiles>[R]NC(=O)C(=S)N/N=C\c1ccccn1</smiles>

16a-r<smiles>[R]NC(=O)c1nnc(-c2ccc(CCNC(=O)C3=NNC(c4ccccn4)S3)cc2)s1</smiles>

17a-r

18a-r

Scheme 4. Reaction of pyridine carboxaldehydes 15a-c with thiohydrazides 14a-m. 
The difference between this reaction and the reaction of oxamic acid thiohydrazides with benzaldehydes described in our previous studies ${ }^{10-12}$ lies in the fact that the latter reaction produces stable hydrazones, which can be transformed into thiadiazoles only after an additional oxidation step. Apparently, the electronwithdrawing nature of pyridine facilitates the formation of the dihydrothiazole moiety followed by oxidation to the thiadiazole ring. Therefore, the one-pot reaction of pyridine carboxaldehyde with oxamic acid thiohydrazide involves the formation of hydrazone, its cyclization to the dihydrothiazole moiety, and oxidation to the fully unsaturated thiadiazole ring with atmospheric oxygen. The best yields of $\mathbf{1 8 g}(\mathbf{8 0} \%)$ were achieved by refluxing pyridine carboxaldehyde with oxamic acid thiohydrazide in methanol or ethanol.

The use of different acids (acetic, sulfuric, $p$-toluenesulfonic acids) as catalysts in the reaction in alcohols did not lead to either an increase in the rate of the formation of thiadiazole or a higher yield of product.

This is a general method, which can be applied to perform the reactions with three isomers of pyridine carboxaldehyde and prepare thiadiazoles containing different substituents in the carbamoyl moiety 18a-r. The yields of thiadiazoles are given in Table 2 . The reaction was performed in ethanol for $12-24 \mathrm{~h}$. The completion of the reaction was monitored by TLC.

Table 2. Yield of thiadiazoles 18a-r

\begin{tabular}{|c|c|c|c|c|}
\hline Entry & $\mathbf{R}$ & $\begin{array}{l}\text { Pyridinyl } \\
\text { group }\end{array}$ & Time (h) & Yield (\%) \\
\hline $18 a$ & $2,4-\mathrm{Cl}_{2}-\mathrm{C}_{6} \mathrm{H}_{3}$ & 4-Py & 12 & 80 \\
\hline $18 b$ & $2,6-(\mathrm{MeO})_{2} \mathrm{C}_{6} \mathrm{H}_{3}$ & 3-Py & 12 & 50 \\
\hline $18 \mathrm{c}$ & $2,4-(\mathrm{MeO}){ }_{2} \mathrm{C}_{6} \mathrm{H}_{3}$ & 2-Py & 24 & 50 \\
\hline $18 d$ & $2,4-\mathrm{Cl}_{2} \mathrm{C}_{6} \mathrm{H}_{3}$ & 3-Py & 12 & 85 \\
\hline $18 \mathrm{e}$ & $4-\mathrm{BrC}_{6} \mathrm{H}_{4}$ & 4-Py & 12 & 74 \\
\hline $18 f$ & $2-\mathrm{CH}_{3} \mathrm{C}_{6} \mathrm{H}_{4}$ & 4-Py & 12 & 44 \\
\hline $18 \mathrm{~g}$ & $\mathrm{C}_{6} \mathrm{H}_{5}$ & 4-Py & 12 & 80 \\
\hline $18 \mathrm{~h}$ & $\mathrm{Ph}$ & 3-Py & 12 & 68 \\
\hline $18 \mathrm{i}$ & 2-Py & 3-Py & 12 & 38 \\
\hline $18 \mathrm{j}$ & $3,5-(\mathrm{MeO})_{2} \mathrm{C}_{6} \mathrm{H}_{3}$ & 4-Py & 12 & 70 \\
\hline $18 k$ & 2-Py & 4-Py & 12 & 68 \\
\hline 181 & $3-\mathrm{Cl}-\mathrm{C}_{6} \mathrm{H}_{4}$ & 4-Py & 12 & 75 \\
\hline $18 m$ & $3-\mathrm{MeOC}_{6} \mathrm{H}_{4}$ & 4-Py & 12 & 56 \\
\hline $18 n$ & 3- $\mathrm{MeOC}_{6} \mathrm{H}_{4}$ & 3-Py & 12 & 54 \\
\hline 180 & $3-\mathrm{FC}_{6} \mathrm{H}_{4}$ & 3-Py & 12 & 69 \\
\hline $18 p$ & $2,5-(\mathrm{MeO})_{2} \mathrm{C}_{6} \mathrm{H}_{3}$ & 3-Py & 12 & 40 \\
\hline $18 q$ & $2,5-(\mathrm{MeO})_{2} \mathrm{C}_{6} \mathrm{H}_{3}$ & 4-Py & 12 & 40 \\
\hline $18 r$ & $2,6-(\mathrm{MeO}){ }_{2} \mathrm{C}_{6} \mathrm{H}_{3}$ & 4-Py & 12 & 40 \\
\hline
\end{tabular}

As can be seen in Table 2, the nature of oxamic acid thiohydrazides has no significant effect on the reaction rate. The rate of the formation of thiadiazole 18c from the 2-pyridinyl isomer is lower than that from the other two isomers due apparently to the slower oxidation step. The formation of 5-pyridin-2-yl-1,3,4thiadiazolecarboxamide $\mathbf{1 8 c}$ is accelerated by bubbling air, which facilitates the oxidation of intermediate dihydrothiadiazole 17c. 


\section{Conclusions}

A convenient general one-pot method with auto-oxidation step for the synthesis of pyridinyl-1,3,4-thiadiazole2-carboxamides was developed based on the reaction of pyridine carboxaldehydes (2,3, or 4 isomers) with the readily available oxamic acid hydrazides. This method allows the synthesis of 1,3,4-thiadiazoles containing different substituents at the 5-position, including the three isomers of pyridyl substituents, without isolation of the hydrazone intermediates.

\section{Experimental Section}

General. The ${ }^{1} \mathrm{H}$ NMR spectra were recorded on a Bruker AM-300 (300 MHz) spectrometer in $\mathrm{CDCl}_{3}$ and DMSO$d_{6} ;{ }^{13} \mathrm{C}$ NMR spectra, on a Bruker AM-300 (75 MHz) spectrometer in $\mathrm{CDCl}_{3}$ and DMSO- $d_{6}$; residual protons and the carbon atom of the solvent were used as the internal standard. Elemental analysis was performed on a Eurovector EA 3000 automated analyzer. The HRMS measurements were carried out on a Bruker micrOTOF II spectrometer using electrospray ionization (ESI). Melting points were measured on a Boetius hot-stage apparatus and are uncorrected. The reaction mixture analysis and purity of the products was controlled by TLC on Merck Silica gel 60 F254 UV-254 plates.

$\alpha$-Chloroacetamides 12a-m were prepared by a standard procedure ${ }^{13}$ from chloroacetyl chloride and appropriate amines in dichloromethane.

The synthesis of thiohydrazides $\mathbf{1 4 g - m}$ was described previously; ${ }^{11,14}$ oxamic acid thiohydrazides 14a-f were synthesized by procedures described in the cited references.

$\mathbf{N}$-(3,5-Dimethoxyphenyl)-2-hydrazinyl-2-thioxoacetamide (14a). Pale yellow crystals (47\%); m.p. $115{ }^{\circ} \mathrm{C} .{ }^{1} \mathrm{H}$ NMR (300 MHz, DMSO-d $\left.d_{6}\right) 10.13(\mathrm{~s}, 1 \mathrm{H}, \mathrm{NH}), 7.06(\mathrm{~s}, 2 \mathrm{H}, \mathrm{Ar}), 6.84\left(\mathrm{~s}, 1 \mathrm{H}, \mathrm{NH}_{2}\right)$,<smiles>COc1cc(NC(=O)C(=S)NN)cc(OC)c1</smiles>
$6.31\left(\mathrm{~s}, 1 \mathrm{H}, \mathrm{NH}_{2}\right), 3.74(\mathrm{~s}, 6 \mathrm{H}, \mathrm{Me}) .{ }^{13} \mathrm{C} \mathrm{NMR}\left(75 \mathrm{MHz}, \mathrm{DMSO}-d_{6}\right) \delta 165.5(\mathrm{C}=\mathrm{S})$, 157.8 (C=O), 154.5, 144.7, 126.6, 111.9, 107.1, 104.2, 57.7 (1C, $\left.\mathrm{CH}_{3}\right), 56.5$ (1C, $\left.\mathrm{CH}_{3}\right) . \mathrm{C}, 47.05 ; \mathrm{H}, 5.13 ; \mathrm{N}, 16.46$. Found: $\mathrm{C}, 47.15 ; \mathrm{H}, 5.25 ; \mathrm{N}, 16.31 \%$. MS (EI): $\mathrm{m} / \mathrm{z}$ (\%) $255\left(\mathrm{M}^{+}+\mathrm{H}, 55\right), 153\left(\mathrm{C}_{8} \mathrm{H}_{11} \mathrm{NO}_{2}^{+}, 100\right)$.

$\mathbf{N}$-(2,4-Dimethoxyphenyl)-2-hydrazinyl-2-thioxoacetamide (14b). Pale yellow crystals (80\%); m.p. $150{ }^{\circ} \mathrm{C} .{ }^{1} \mathrm{H}$<smiles>COc1ccc(NC(=O)C(=S)NN)c(OC)c1</smiles>
NMR (300 MHz, DMSO-d $\left.d_{6}\right) \delta 10.24(\mathrm{~s}, 1 \mathrm{H}), 8.15(\mathrm{~d}, J=8.5 \mathrm{~Hz}, 1 \mathrm{H}), 7.95(\mathrm{~s}, 1 \mathrm{H})$, $6.70(\mathrm{~s}, 1 \mathrm{H}), 6.57(\mathrm{dd}, J=9.5,1.1 \mathrm{~Hz}, 1 \mathrm{H}), 4.97(\mathrm{~s}, 1 \mathrm{H}), 3.90(\mathrm{~s}, 3 \mathrm{H}), 3.76(\mathrm{~s}, 3 \mathrm{H}) .{ }^{13} \mathrm{C}$ NMR $\left(75 \mathrm{MHz}, \mathrm{DMSO}-d_{6}\right) \delta 166.5(\mathrm{C}=\mathrm{S}), 158.9(\mathrm{C}=0), 153.5,148.5,123.6,112.8$, 108.7, 104.1, 56.0(1C, $\left.\mathrm{CH}_{3}\right), 55.7\left(1 \mathrm{C}, \mathrm{CH}_{3}\right)$. Calcd for $\mathrm{C}_{10} \mathrm{H}_{13} \mathrm{~N}_{3} \mathrm{O}_{3} \mathrm{~S}: \mathrm{C}, 47.05 ; \mathrm{H}$, 5.13; N, 16.46. Found: $\mathrm{C}, 47.22 ; \mathrm{H}, 5.20 ; \mathrm{N}, 16.21 \%$. MS (EI): $m / z(\%) 255\left(\mathrm{M}^{+}+\mathrm{H}\right.$,

64), $153\left(\mathrm{C}_{8} \mathrm{H}_{11} \mathrm{NO}_{2}^{+}, 100\right)$.

$\mathbf{N}$-(2,5-Dimethoxyphenyl)-2-hydrazinyl-2-thioxoacetamide (14c). Pale yellow crystals (77\%); m.p. $120{ }^{\circ} \mathrm{C} .{ }^{1} \mathrm{H}$

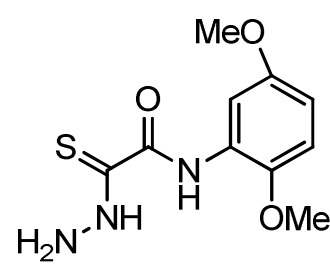
NMR (300 MHz, DMSO-d $\left.)_{6}\right) \delta 10.44(s, 1 \mathrm{H}), 7.94(\mathrm{~d}, J=3.1 \mathrm{~Hz}, 1 \mathrm{H}), 7.04(\mathrm{~d}, J=9.0 \mathrm{~Hz}$, $1 \mathrm{H}), 6.77-6.65(\mathrm{~m}, 1 \mathrm{H}), 3.86(\mathrm{~s}, 3 \mathrm{H}), 3.71(\mathrm{~s}, 3 \mathrm{H}) .{ }^{13} \mathrm{C}$ NMR $\left(75 \mathrm{MHz}, \mathrm{DMSO}-d_{6}\right) \delta 168.5$ $(\mathrm{C}=\mathrm{S}), 157.8(\mathrm{C}=\mathrm{O}), 153.5,142.7,127.6,111.9,108.1,106.3,56.7\left(1 \mathrm{C}, \mathrm{CH}_{3}\right), 55.7$ (1C, $\mathrm{CH}_{3}$ ). Calcd for $\mathrm{C}_{10} \mathrm{H}_{13} \mathrm{~N}_{3} \mathrm{O}_{3} \mathrm{~S}: \mathrm{C}, 47.05 ; \mathrm{H}, 5.13 ; \mathrm{N}, 16.46$. Found: $\mathrm{C}, 47.18 ; \mathrm{H}, 5.19 ; \mathrm{N}$, $16.51 \% . \mathrm{MS}(\mathrm{EI}): \mathrm{m} / \mathrm{z}(\%) 255\left(\mathrm{M}^{+}+\mathrm{H}, 50\right), 153\left(\mathrm{C}_{8} \mathrm{H}_{11} \mathrm{NO}_{2}{ }^{+}, 100\right)$.

$\mathbf{N}$-(3,4-Dimethoxyphenyl)-2-hydrazinyl-2-thioxoacetamide (14d). Pale yellow crystals (77\%); m.p. $135{ }^{\circ} \mathrm{C} .{ }^{1} \mathrm{H}$ NMR (300 MHz, DMSO-d $\left.d_{6}\right) \delta 10.07$ (s, 1H, NH), $9.04(\mathrm{~s}, 1 \mathrm{H}, \mathrm{NH}), 7.95\left(\mathrm{~s}, 1 \mathrm{H}, \mathrm{NH}_{2}\right), 7.41$ 
<smiles>COc1ccc(NC(=O)C(=S)NN)cc1OC</smiles>

(d, J = $1.9 \mathrm{~Hz}, 1 \mathrm{H}, \mathrm{Ar}), 7.30(\mathrm{~s}, 1 \mathrm{H}, \mathrm{Ar}), 6.89(\mathrm{~m}, 1 \mathrm{H}, \mathrm{Ar}), 5.86\left(\mathrm{~s}, 1 \mathrm{H}, \mathrm{NH}_{2}\right), 2.89(\mathrm{~s}, 3 \mathrm{H}$, $\left.\mathrm{CH}_{3}\right), 2.73\left(\mathrm{~s}, 3 \mathrm{H}, \mathrm{CH}_{3}\right) .{ }^{13} \mathrm{C} \mathrm{NMR}\left(75 \mathrm{MHz}, \mathrm{DMSO}-d_{6}\right) \delta 166.4(\mathrm{C}=\mathrm{S}), 156.8(\mathrm{C}=\mathrm{O})$, 154.5, 145.7, 127.6, 111.9, 110.1, 105.4, 57.7 (1C, $\left.\mathrm{CH}_{3}\right), 56.2$ (1C, $\mathrm{CH}_{3}$ ). Calcd for $\mathrm{C}_{10} \mathrm{H}_{13} \mathrm{~N}_{3} \mathrm{O}_{3} \mathrm{~S}: \mathrm{C}, 47.05 ; \mathrm{H}, 5.13 ; \mathrm{N}, 16.46$. Found: $\mathrm{C}, 46.95 ; \mathrm{H}, 5.25 ; \mathrm{N}, 16.55 \%$. MS (EI): $\mathrm{m} / \mathrm{z}(\%) 255\left(\mathrm{M}^{+}+\mathrm{H}, 60\right), 153\left(\mathrm{C}_{8} \mathrm{H}_{11} \mathrm{NO}_{2}{ }^{+}, 100\right)$.

$\mathbf{N}$-(2,6-Dimethoxyphenyl)-2-hydrazinyl-2-thioxoacetamide (14e). Pale yellow crystals (38\%); m.p. $175^{\circ} \mathrm{C} .{ }^{1} \mathrm{H}$ NMR (300 MHz, DMSO-d $\left.d_{6}\right) \delta 9.68$ (s, 1H), $9.49(\mathrm{~s}, \mathrm{H}), 9.03$ $(\mathrm{s}, 1 \mathrm{H}), 7.95(\mathrm{~s}, 1 \mathrm{H}), 5.99(\mathrm{~s}, 2 \mathrm{H}), 2.52\left(\mathrm{~s}, 6 \mathrm{H}, \mathrm{CH}_{3}\right) .{ }^{13} \mathrm{C}$ NMR $\left(75 \mathrm{MHz}, \mathrm{DMSO}-d_{6}\right) \delta 167.0$ $(\mathrm{C}=\mathrm{S}), 157.6(\mathrm{C}=\mathrm{O}), 155.8(2 \mathrm{C}), 128.6,113.8,104.9(2 \mathrm{C}), 56.3\left(2 \mathrm{C}, \mathrm{CH}_{3}\right)$. Calcd for $\mathrm{C}_{10} \mathrm{H}_{13} \mathrm{~N}_{3} \mathrm{O}_{3} \mathrm{~S}: \mathrm{C}, 47.05 ; \mathrm{H}, 5.13 ; \mathrm{N}, 16.46$. Found: $\mathrm{C}, 47.21 ; \mathrm{H}, 5.28 ; \mathrm{N}, 16.34 \%$. MS (EI): $\mathrm{m} / \mathrm{z}(\%) 255\left(\mathrm{M}^{+}+\mathrm{H}, 72\right), 153\left(\mathrm{C}_{8} \mathrm{H}_{11} \mathrm{NO}_{2}{ }^{+}, 100\right)$.

N-(2,4-Dichlorophenyl-2-hydrazinyl-2-thioxoacetamide (14f). White crystals (87\%); m.p. $190{ }^{\circ} \mathrm{C} .{ }^{1} \mathrm{H}$ NMR $(300$<smiles>NNC(=S)C(=O)Nc1ccc(Cl)cc1Cl</smiles>
$\left.\mathrm{MHz}, \mathrm{DMSO}-\mathrm{d}_{6}\right) \delta 10.42(\mathrm{~s}, 1 \mathrm{H}), 8.12(\mathrm{~s}, 1 \mathrm{H}), 7.75(\mathrm{~d}, J=8.9 \mathrm{~Hz}, 1 \mathrm{H}), 7.56(\mathrm{~d}, J=8.8 \mathrm{~Hz}$, 1H). ${ }^{13} \mathrm{C}$ NMR (75 MHz, DMSO-d $\left.d_{6}\right) \delta 159.6$ (C=S), 159.2 (C=O), 138.2, 131.4, 131.0, 126.5, 122.1, 120.9. Calcd for $\mathrm{C}_{8} \mathrm{H}_{7} \mathrm{Cl}_{2} \mathrm{~N}_{3} \mathrm{OS}$ : C, 36.38; $\mathrm{H}, 2.67 ; \mathrm{N}, 15.91$. Found: $\mathrm{C}, 36.40$; $\mathrm{H}, 2.60 ; \mathrm{N}, 15.8 \%$.

\section{General procedure for the synthesis of thiadiazoles 18a-r}

Pyridine carboxaldehydes $15(0.5 \mathrm{mmol})$ was added dropwise to a hot solution of thiohydrazide 14 (0.5 mmol) in ethanol $(20 \mathrm{~mL})$. The mixture was refluxed for $24 \mathrm{~h}$ in open atmosphere. After completion of the reaction (TLC monitoring; $\mathrm{CH}_{2} \mathrm{Cl}_{2}: \mathrm{EtOH}, 9: 1$ ), the reaction mixture was allowed to stand at room temperature. The formed precipitate was filtered off and washed with cold ethanol. For compound $\mathbf{1 8 \mathbf { j }}$, the reaction mixture was bubbled with atmospheric air at room temperature for $24 \mathrm{~h}$, and then the formed precipitate was filtered off.

$\mathbf{N}$-(2,4-Dichlorophenyl)-5-(pyridin-4-yl)-1,3,4-thiadiazole-2-carboxamide (18a). Colorless crystals (80\%); m.p.

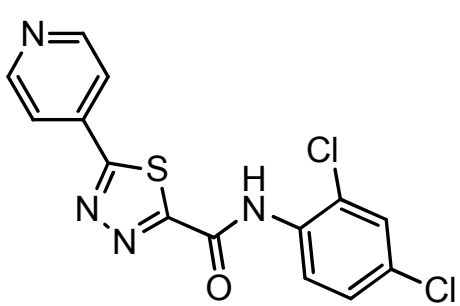
$100{ }^{\circ} \mathrm{C} .{ }^{1} \mathrm{H}$ NMR (300 MHz, DMSO- $\left.d_{6}\right) \delta 11.54(\mathrm{~s}, 1 \mathrm{H}, \mathrm{NH}), 8.83(\mathrm{~d}, J=5.8 \mathrm{~Hz}, 2 \mathrm{H}$, Py), 8.21 (d, J = 2.2 Hz, 1H, Ar), 8.04 (d, J = 5.9 Hz, 2H, Py), 7.88 (dd, J = 8.8, $2.3 \mathrm{~Hz}$, $1 \mathrm{H}, \mathrm{Ar}), 7.66(\mathrm{~d}, J=8.9 \mathrm{~Hz}, 1 \mathrm{H}, \mathrm{Ar}) .{ }^{13} \mathrm{C} \mathrm{NMR}\left(75 \mathrm{MHz}, \mathrm{DMSO}-d_{6}\right) \delta 170.5$ (C, thiadiazole), 166.8 (1C, C=O), 156.6 (C,thiadiazole), 151.4 (2C, Py), 138.2 (1C, Ar), 136.2 (1C, Py), 131.4 (1C, Ar), 131.1 (2C, Py), 126.9 (1C, Ar), 122.5 (1C, Ar), 122.1 (1C, Ar), 121.2 (1C, Ar). HRMS (ESI) $\mathrm{C}_{14} \mathrm{H}_{10} \mathrm{Cl}_{2} \mathrm{~N}_{4} \mathrm{OS}$. Calcd for $(\mathrm{M}+\mathrm{H})^{+}: 350.9869$. Found: 350.9865.

N-(2,6-Dimethoxyphenyl)-5-(pyridin-3-yl)-1,3,4-thiadiazole-2-carboxamide (18b). Colorless crystals (50\%); m.p. $170{ }^{\circ} \mathrm{C} .{ }^{1} \mathrm{H}$ NMR $\left(300 \mathrm{MHz} \mathrm{CDCl}_{3}\right) \delta 9.25$ (s, 1H, NH), 8.81 (d, J = 4.4 Hz, 1H, Py), 8.58 (s, 1H, Py), 8.38 (d, J

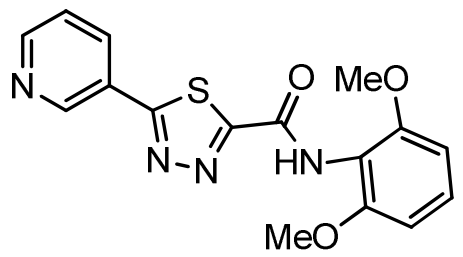
$=7.9 \mathrm{~Hz}, 1 \mathrm{H}, \mathrm{Py}), 7.51(\mathrm{dd}, J=7.7,5.0 \mathrm{~Hz}, 1 \mathrm{H}, \mathrm{Ar}), 7.31(\mathrm{dd}, J=7.7,5.0 \mathrm{~Hz}, 1 \mathrm{H})$, $6.67(\mathrm{~d}, J=8.4 \mathrm{~Hz}, 2 \mathrm{H}, \mathrm{Ar}), 3.89\left(\mathrm{~s}, 6 \mathrm{H}, \mathrm{CH}_{3}\right) .{ }^{13} \mathrm{C} \mathrm{NMR}\left(75 \mathrm{MHz}, \mathrm{CDCl}_{3}\right) \delta 169.4(\mathrm{C}$, thiadiazole), 165.69 (1C, C=0), 155.4 (C, thiadiazole), 152.3 (1C, Py), 148.8 (1C, Py), 135.2 (1C, Py), 128.5 (2C, Ar), 126.1 (1C, Py), 124.0 (1C, Ar), 123.4 (1C, Py), 112.5 (1C, Ar), 104.3 (2C, Ar), $56.0\left(2 \mathrm{C}, \mathrm{CH}_{3}\right)$. HRMS (ESI) $\mathrm{C}_{16} \mathrm{H}_{14} \mathrm{~N}_{4} \mathrm{O}_{3} \mathrm{~S}$. Calcd for $(\mathrm{M}+\mathrm{H})^{+}:$343.0859. Found: 343.0858 .

N-(2,4-Dimethoxyphenyl)-5-(pyridin-2-yl)-1,3,4-thiadiazole-2-carboxamide (18c). Yellow crystals (50\%); m.p. $170{ }^{\circ} \mathrm{C} .{ }^{1} \mathrm{H}$ NMR $\left(300 \mathrm{MHz}, \mathrm{CDCl}_{3}\right) \delta 9.62(\mathrm{~s}, 1 \mathrm{H}$, $\mathrm{NH}), 9.26$ (d, $J=1.5 \mathrm{~Hz}, 1 \mathrm{H}, \mathrm{Py}), 8.81$ (d, $J=5.9 \mathrm{~Hz}, 1 \mathrm{H}, \mathrm{Py}), 8.37$ (d, $J=9.7 \mathrm{~Hz}$, $2 \mathrm{H}, \mathrm{Py}), 7.51$ (dd, J = 8.0, 5.0 Hz, 1H, Ar), $6.59-6.52(\mathrm{~m}, 2 \mathrm{H}, \mathrm{Ar}), 3.96\left(\mathrm{~s}, 3 \mathrm{H}, \mathrm{CH}_{3}\right)$, $3.85\left(\mathrm{~s}, 3 \mathrm{H}, \mathrm{CH}_{3}\right) .{ }^{13} \mathrm{C} \mathrm{NMR}\left(75 \mathrm{MHz}, \mathrm{DMSO}-d_{6}\right)$ low solubility. HRMS (ESI) 
$\mathrm{C}_{16} \mathrm{H}_{14} \mathrm{~N}_{4} \mathrm{O}_{3} \mathrm{~S}$. Calcd for $(\mathrm{M}+\mathrm{H})^{+}:$343.0859. Found: 343.0854 .

N-(2,4-Dichlorophenyl)-5-(pyridin-3-yl)-1,3,4-thiadiazole-2-carboxamide (18d). Colorless crystals (85\%); m.p. $205{ }^{\circ} \mathrm{C} .{ }^{1} \mathrm{H}$ NMR $\left(300 \mathrm{MHz}, \mathrm{CDCl}_{3}\right) \delta 9.26(\mathrm{~s}, 1 \mathrm{H}, \mathrm{NH}), 9.15$ (s, $\left.1 \mathrm{H}, \mathrm{Py}\right), 8.83$

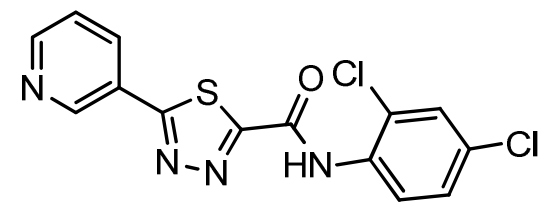
(m, 1H,Py), 8.38 (d, J = 7.0 Hz, 1H,Py), 8.01 (s, 1H, Ar), 7.61-7.46 (m, 3H, Ar+Py). ${ }^{13} \mathrm{C}$ NMR (75 MHz, DMSO- $\left.d_{6}\right) \delta 170.0$ (C, thiadiazole), 167.2 (1C, $\mathrm{C}=0$ ), 166.9 (C, thiadiazole), 156.0 (1C, Py), 152.2 (1C,Py), 148.1 (1C, Py), 136.2 (1C, Ar), 137.2 (1C, Py), 131.4 (1C, Ar), 130.1 (1C, Py), 125.9 (1C, Ar), 123.2 (1C, Ar), 122.1 (1C, Ar), 120.2 (1C, Ar). HRMS (ESI) $\mathrm{C}_{14} \mathrm{H}_{10} \mathrm{Cl}_{2} \mathrm{~N}_{4} \mathrm{OS}$. Calcd for $(\mathrm{M}+\mathrm{H})^{+}:$350.9869. Found: 350.9865.

N-(4-Bromophenyl)-5-(pyridin-4-yl)-1,3,4-thiadiazole-2-carboxamide (18e). Colorless crystals (74\%); m.p.

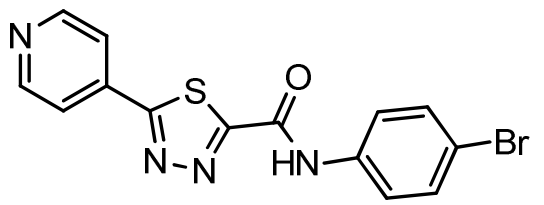
$235{ }^{\circ} \mathrm{C} .{ }^{1} \mathrm{H}$ NMR $\left(300 \mathrm{MHz}, \mathrm{CDCl}_{3}\right) \delta 9.15(\mathrm{~s}, 1 \mathrm{H}, \mathrm{NH}), 8.87$ (d, J = $5.5 \mathrm{~Hz}, 2 \mathrm{H}$, Py), 7.92 (d, J = 5.6 Hz, 2H, Py), 7.65 (d, J = 8.8 Hz, 2H, Ar), 7.56 (d, J=8.8 Hz, $2 \mathrm{H}, \mathrm{Ar}) .{ }^{13} \mathrm{C}$ NMR (75 MHz, DMSO- $\left.d_{6}\right) \delta 170.4$ (C, thiadiazole), $167.2(1 \mathrm{C}, \mathrm{C}=\mathrm{O})$, 156.4 (C, thiadiazole), 151.4 (2C, Py), 137.4 (1C,Py), 136.3 (1C,Ar), 132.1 (2C, Py), 123.2 (2C, Ar), 122.20 (2C, Ar), 117.25 (1C, Ar). HRMS (ESI) $\mathrm{C}_{14} \mathrm{H}_{9} \mathrm{BrN}_{4} \mathrm{OS}$. Calcd for $(\mathrm{M}+\mathrm{H})^{+}: 360.9753$. Found: 360.9743 .

5-(Pyridin-4-yl)-N-(o-tolyl)-1,3,4-thiadiazole-2-carboxamide (18f). Colorless crystals (44\%); m.p. $160{ }^{\circ} \mathrm{C} .{ }^{1} \mathrm{H}$

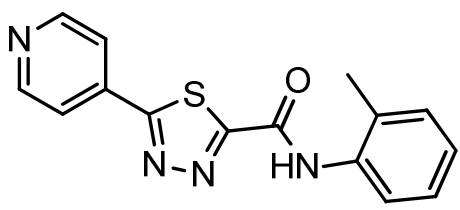
$\operatorname{NMR}\left(300 \mathrm{MHz}, \mathrm{CDCl}_{3}\right) \delta 9.12(\mathrm{~s}, 1 \mathrm{H}, \mathrm{NH}), 8.86$ (d, $\left.J=5.7 \mathrm{~Hz}, 2 \mathrm{H}, \mathrm{Py}\right), 8.12$ (d, J = 8.0 $\mathrm{Hz}, 1 \mathrm{H}, \mathrm{Ar}$ ), 7.91 (d, J = 5.9 Hz, 2H,Py), 7.33 (d, $J=7.7 \mathrm{~Hz}, 1 \mathrm{H}, \mathrm{Ar}), 7.19$ (t, $J=7.4 \mathrm{~Hz}$, $1 \mathrm{H}, \mathrm{Ar}), 2.45\left(\mathrm{~s}, 3 \mathrm{H}, \mathrm{CH}_{3}\right) \cdot{ }^{13} \mathrm{C}$ NMR $\left(75 \mathrm{MHz}, \mathrm{DMSO}-d_{6}\right) \delta 156.4(1 \mathrm{C}, \mathrm{C}=0), 151.4$ (C, thiadiazole), 151.0 (C, thiadiazole), 136.4 (2C, Py), 135.2 (2C, Py), 134.0 (1C, Ar), 130.9 (1C, Py), 127.2 (1C, Ar), 126.8 (1C, Ar), 126.6 (1C, Ar), 123.1 (1C, Ar), 122.2 (1C, Ar), $18.49\left(1 \mathrm{C}, \mathrm{CH}_{3}\right)$. HRMS (ESI) $\mathrm{C}_{15} \mathrm{H}_{12} \mathrm{~N}_{4} \mathrm{OS}$. Calcd for $(\mathrm{M}+\mathrm{H})^{+}:$297.0805. Found: 290.0801.

N-Phenyl-5-(pyridin-4-yl)-1,3,4-thiadiazole-2-carboxamide (18g). White crystals (80\%); m.p. $210-212{ }^{\circ} \mathrm{C} .{ }^{1} \mathrm{H}$ NMR (300 MHz, DMSO- $\left.d_{6}\right) \delta 11.28(\mathrm{~s}, 1 \mathrm{H}, \mathrm{NH}), 8.83(\mathrm{~d}, J=5.6 \mathrm{~Hz}, 2 \mathrm{H}, \mathrm{Py}), 8.05$

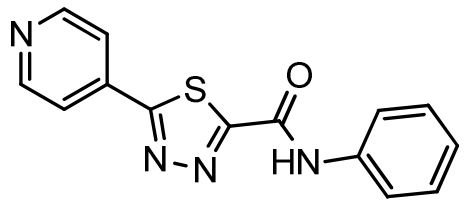
(d, J=5.7 Hz, 2H, Py), 7.87 (d, J = 7.9 Hz, 2H, Ar), 7.40 (t, J = 7.8 Hz, 2H, Ar), 7.19 $(t, J=7.3 \mathrm{~Hz}, 1 \mathrm{H}, \mathrm{Ar}) .{ }^{13} \mathrm{C}$ NMR (75 MHz, DMSO- $\left.d_{6}\right) \delta 170.3$ (C, thiadiazole), 167.4 (1C, C=O), 156.3 (C, thiadiazole), 151.4 (2C, Py), 138.0 (1C, Py), 136.4 (1C, Ar), 129.2 (2C, Py), 125.3 (1C, Ar), 122.1 (2C, Ar), 121.3 (2C, Ar). HRMS (ESI) $\mathrm{C}_{14} \mathrm{H}_{10} \mathrm{~N}_{4} \mathrm{OS}$. Calcd for $(\mathrm{M}+\mathrm{H})^{+}: 283.0648$. Found: 283.0646.

N-Phenyl-5-(pyridin-3-yl)-1,3,4-thiadiazole-2-carboxamide (18h). White crystals (60\%); m.p. $190-192{ }^{\circ} \mathrm{C} .{ }^{1} \mathrm{H}$

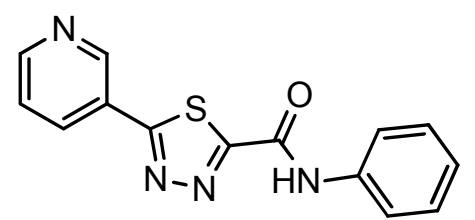
NMR (300 MHz, DMSO-d $\left.d_{6}\right) 11.27(\mathrm{~s}, 1 \mathrm{H}, \mathrm{NH}), 9.28(\mathrm{~s}, 1 \mathrm{H}, \mathrm{Py}), 8.82(\mathrm{~d}, J=4.8 \mathrm{~Hz}$, $1 \mathrm{H}, \mathrm{Py}), 8.50$ (dd, $J=4.4,3.7 \mathrm{~Hz}, 1 \mathrm{H}, \mathrm{Py}), 7.88$ (d, $J=7.9 \mathrm{~Hz}, 2 \mathrm{H}, \mathrm{Ar}), 7.66$ (dd, $J=$ 7.9, $4.9 \mathrm{~Hz}, 1 \mathrm{H}, \mathrm{Py}), 7.41(\mathrm{t}, J=7.8 \mathrm{~Hz}, 2 \mathrm{H}, \mathrm{Ar}), 7.19(\mathrm{t}, J=7.2 \mathrm{~Hz}, 1 \mathrm{H}, \mathrm{Ar}) .{ }^{13} \mathrm{CNMR}$ (75 MHz, DMSO- $\left.d_{6}\right) \delta 169.5$ (C, thiadiazole), 166.7 (C, thiadiazole), $166.6(\mathrm{C}=0)$, 156.4 (1C,Py), 152.9 (1C, Py), 148.9 (1C, Py), 138.0 (1C, Py), 135.9 (1C, Ar), 129.2 (1C, Py), 125.2 (2C, Ar), 124.8 (1C, Ar), 121.3 (2C, Ar). HRMS (ESI) $\mathrm{C}_{14} \mathrm{H}_{10} \mathrm{~N}_{4} \mathrm{OS}$. Calcd for (M+H) ${ }^{+}: 283.0648$. Found: 283.0646.

N-(Pyridin-2-yl)-5-(pyridin-3-yl)-1,3,4-thiadiazole-2-carboxamide (18i). White crystals (38\%); m.p. $180-182{ }^{\circ} \mathrm{C}$.

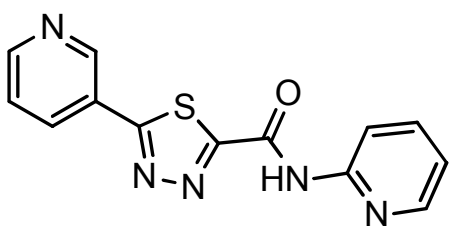

${ }^{1} \mathrm{H}$ NMR (300 MHz, CDCl 3 ) $\delta 9.71(\mathrm{~s}, 1 \mathrm{H}, \mathrm{NH}), 9.02\left(\mathrm{~s}, 1 \mathrm{H}, \mathrm{Py}^{\prime}\right), 8.82(\mathrm{~d}, J=4.1 \mathrm{~Hz}$, $1 \mathrm{H}, \mathrm{Py}^{\prime}$ ), 8.23 (d, J=8.3 Hz, 1H, Py'), 7.92 (d, J=7.8 Hz, 1H, Py), 7.82 (t, J= $7.4 \mathrm{~Hz}$, $1 \mathrm{H}, \mathrm{Py}), 7.52$ (dd, J = 7.8, 4.9 Hz, 1H, Py'), 7.39 (dd, $J=7.7,5.0 \mathrm{~Hz}, 1 \mathrm{H}, \mathrm{Py}), 7.12-$ $7.03(\mathrm{~m}, 1 \mathrm{H}, \mathrm{Py}) .{ }^{13} \mathrm{C}$ NMR $\left(75 \mathrm{MHz}, \mathrm{CDCl}_{3}\right) \delta 171.9$ (C, thiadiazole), $165.8(\mathrm{C}=\mathrm{O})$, 153.4 (C, thiadiazole), 156.6 (1C, Py), 151.1 (1C, Py), 147.9 (1C, Py), 148.5 (1C, Py), 
138.0 (1C, Py), 136.3 (1C, Py), 129.0 (1C, Py) 121.4 (1C, Py), 120.9 (1C, Py), 113.9 (1C, Py). HRMS (ESI) $\mathrm{C}_{13} \mathrm{H}_{9} \mathrm{~N}_{5} \mathrm{OS}$. Calcd for $(\mathrm{M}+\mathrm{H})^{+}:$284.0601. Found: 284.0605 .

N-(3,5-Dimethoxyphenyl)-5-(pyridin-4-yl)-1,3,4-thiadiazole-2-carboxamide (18j). Yellow crystals (70\%); m.p.<smiles>COc1cc(NC(=O)c2nnc(-c3ccncc3)s2)cc(OC)c1</smiles>
179-180 ${ }^{\circ} \mathrm{C} .{ }^{1} \mathrm{H}$ NMR $\left(300 \mathrm{MHz}, \mathrm{CDCl}_{3}\right) \delta 9.19(\mathrm{~s}, 1 \mathrm{H}, \mathrm{NH}), 8.96(\mathrm{~d}, J=4.1 \mathrm{~Hz}$, $2 \mathrm{H}, \mathrm{Py}), 8.06-7.97(\mathrm{~m}, 2 \mathrm{H}, \mathrm{Py}), 7.38(\mathrm{~s}, 1 \mathrm{H}, \mathrm{Ar}), 7.06(\mathrm{~d}, J=2.0 \mathrm{~Hz}, 1 \mathrm{H}, \mathrm{Ar})$, 6.47 (d, J = $1.9 \mathrm{~Hz}, 1 \mathrm{H}, \mathrm{Ar}), 3.95\left(\mathrm{~s}, 6 \mathrm{H}, \mathrm{CH}_{3}\right) .{ }^{13} \mathrm{C} \mathrm{NMR}\left(75 \mathrm{MHz}, \mathrm{DMSO}-d_{6}\right)$ low solubility. HRMS (ESI) $\mathrm{C}_{16} \mathrm{H}_{14} \mathrm{~N}_{4} \mathrm{O}_{3} \mathrm{~S}$. Calcd for $(\mathrm{M}+\mathrm{H})^{+}$: 343.0859. Found: 343.0850 .

N-(Pyridin-2-yl)-5-(pyridin-4-yl)-1,3,4-thiadiazole-2-carboxamide (18k). White crystals (68\%); m.p. $190-192{ }^{\circ} \mathrm{C}$.

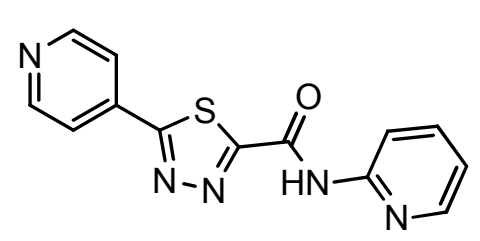
${ }^{1} \mathrm{H}$ NMR $\left(300 \mathrm{MHz} \mathrm{CDCl}_{3}\right) \delta 9.73(\mathrm{~s}, 1 \mathrm{H}, \mathrm{NH}), 8.86(\mathrm{~d}, J=5.3 \mathrm{~Hz}, 2 \mathrm{H}, \mathrm{Py}$ ), 8.43 (d, J $\left.=4.1 \mathrm{~Hz}, 1 \mathrm{H}, \mathrm{Py}), 8.32(\mathrm{~d}, J=8.3 \mathrm{~Hz}, 1 \mathrm{H}, \mathrm{Py}), 7.92(\mathrm{~d}, J=5.3 \mathrm{~Hz}, 2 \mathrm{H}, \mathrm{Py})^{\prime}\right), 7.82(\mathrm{t}, J=$ $\left.7.8 \mathrm{~Hz}, 1 \mathrm{H}, \mathrm{Py}), 7.23-7.13(\mathrm{~m}, 1 \mathrm{H}, \mathrm{Py}) .{ }^{13} \mathrm{C} \mathrm{NMR}\left(75 \mathrm{MHz}^{\mathrm{CDCl}}\right)_{3}\right) \delta 170.9(\mathrm{C}$, thiadiazole), 165.6 ( $\mathrm{C}=\mathrm{O}), 155.4$ (C, thiadiazole), 151.1 (2C, Py), 149.9 (1C, Py), 148.5 (1C, Py), 138.5 (2C, Py'), 136.3 (1C, Py), 121.6 (1C, Py), 120.9 (1C, Py), 114.3 (1C, Py). HRMS (ESI) $\mathrm{C}_{13} \mathrm{H}_{9} \mathrm{~N}_{5} \mathrm{OS}$. Calcd for $(\mathrm{M}+\mathrm{H})^{+}: 284.0601$. Found: 284.0600.

$\mathbf{N}$-(3-Chlorophenyl)-5-(pyridin-4-yl)-1,3,4-thiadiazole-2-carboxamide (18I). Colorless crystals (75\%); m.p. 175$177{ }^{\circ} \mathrm{C} .{ }^{1} \mathrm{H}$ NMR $\left(300 \mathrm{MHz}, \mathrm{CDCl}_{3}\right) \delta 9.16(\mathrm{~s}, 1 \mathrm{H}, \mathrm{NH}), 8.86(\mathrm{~d}, J=5.8 \mathrm{~Hz}, 2 \mathrm{H}, \mathrm{Py})$,

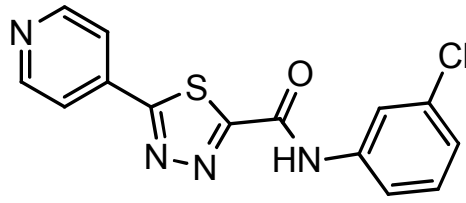

$7.95-7.87(\mathrm{~m}, 3 \mathrm{H}, \mathrm{Py}+\mathrm{Ar}), 7.56(\mathrm{~d}, J=9.1 \mathrm{~Hz}, 1 \mathrm{H}, \mathrm{Py}), 7.36(\mathrm{t}, J=8.1 \mathrm{~Hz}, 1 \mathrm{H}, \mathrm{Py})$, $7.22(\mathrm{~d}, J=8.5 \mathrm{~Hz}, 1 \mathrm{H}, \mathrm{Ar}) .{ }^{13} \mathrm{C} \mathrm{NMR}\left(75 \mathrm{MHz}, \mathrm{CDCl}_{3}\right) \delta 165.8$ (C=O), 155.0 (C, thiadiazole), 151.1 (2C, Py)u, 137.4 (C, thiadiazole), 136.2 (2C, Py), 135.0 (1C, Py), 130.3 (1C, Ar), 125.6 (1C, Ar), 121.6 (1C, Ar), 120.1 (1C, Ar), 118.0 (1C, Ar), 115.7 (1C, Ar). HRMS (ESI) $\mathrm{C}_{14} \mathrm{H}_{9} \mathrm{CIN}_{4} \mathrm{OS}$. Calcd for $(\mathrm{M}+\mathrm{H})^{+}:$317.0258. Found: 317.0248.

$\mathbf{N}$-(3-Methoxyphenyl)-5-(pyridin-4-yl)-1,3,4-thiadiazole-2-carboxamide (18m). Colorless crystals (56\%); m.p.

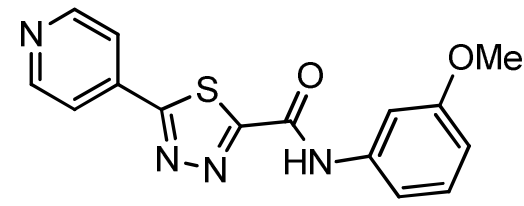
$150-153{ }^{\circ} \mathrm{C} .{ }^{1} \mathrm{H}$ NMR $\left(300 \mathrm{MHz}, \mathrm{CDCl}_{3}\right) \delta 9.15-9.03(\mathrm{~m}, 1 \mathrm{H}, \mathrm{NH}), 8.90-8.81$ $(\mathrm{m}, 2 \mathrm{H}, \mathrm{Py}), 7.92(\mathrm{~d}, J=6.0 \mathrm{~Hz}, 2 \mathrm{H}, \mathrm{Py}), 7.47$ (s, 1H, Ar), 7.34 (t, J = $8.1 \mathrm{~Hz}$, $1 \mathrm{H}, \mathrm{Ar}), 7.22$ (d, J = $7.9 \mathrm{~Hz}, 1 \mathrm{H}, \mathrm{Ar}), 6.81(\mathrm{~d}, J=7.9 \mathrm{~Hz}, 1 \mathrm{H}, \mathrm{Ar}), 3.88(\mathrm{~s}, 3 \mathrm{H}$, $\mathrm{CH}_{3}$ ). ${ }^{13} \mathrm{C}$ NMR (75 MHz, DMSO- $\left.d_{6}\right) \delta 170.7$ (C, thiadiazole), 167.7 (C=0), 166.8 (C, thiadiazole), 152.1 (2C, Py), 148.9 (1C, Py), 139.0 (1C, Ar), 125.9 (2C, Py), 130.0 (1C, Ar), 113.6 (1C, Ar), 110.9 (1C, Ar), 106.2 (1C, Ar), 104.2 (1C, Ar), 57.5 (CH3). HRMS (ESI) $\mathrm{C}_{15} \mathrm{H}_{12} \mathrm{~N}_{4} \mathrm{O}_{2} \mathrm{~S}$. Calcd for $(\mathrm{M}+\mathrm{H})^{+}: 313.0754$. Found: 313.0762 .

N-(3-Methoxyphenyl)-5-(pyridin-3-yl)-1,3,4-thiadiazole-2-carboxamide (18n). Colorless crystals (54\%); m.p. 145-147 ${ }^{\circ} \mathrm{C} .{ }^{1} \mathrm{H}$ NMR (300 MHz, $\left.\mathrm{CDCl}_{3}\right) \delta 9.26(\mathrm{~s}, 1 \mathrm{H}, \mathrm{NH}), 9.10(\mathrm{~m}, 2 \mathrm{H}, \mathrm{Py})$,

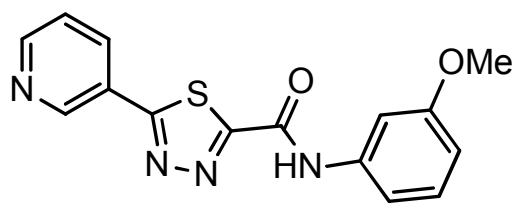
$8.82(\mathrm{~d}, J=3.8 \mathrm{~Hz}, 1 \mathrm{H}, \mathrm{Py}), 8.38(\mathrm{~d}, J=7.8 \mathrm{~Hz}, 1 \mathrm{H}, \mathrm{Py}), 7.47(\mathrm{~s}, 1 \mathrm{H}, \mathrm{Ar}), 7.34$ (t, $J=8.2 \mathrm{~Hz}, 1 \mathrm{H}, \mathrm{Ar}), 7.22(\mathrm{~d}, J=7.9 \mathrm{~Hz}, 1 \mathrm{H}, \mathrm{Ar}), 6.80(\mathrm{~d}, J=8.2 \mathrm{~Hz}, 1 \mathrm{H}, \mathrm{Ar}), 3.88$ $\left(\mathrm{s}, 3 \mathrm{H}, \mathrm{CH}_{3}\right) .{ }^{13} \mathrm{C}$ NMR (75 MHz, DMSO-d 6 ) $\delta 169.7$ (C, thiadiazole), 166.7 (C=O), 159.8 (C, thiadiazole), 156.1 (1C, Py), 153.0 (1C, Py), 148.9(1C, Py), 139.0(1C, Ar), 135.9 (1C, Py), 130.0 (1C, Ar), 124.8 (1C, Py), 113.6 (1C, Ar), 110.9 (1C, Ar), 107.2 (1C, Ar), 105.5 (1C, Ar), $55.5\left(\mathrm{CH}_{3}\right)$. HRMS (ESI) $\mathrm{C}_{15} \mathrm{H}_{12} \mathrm{~N}_{4} \mathrm{O}_{2} \mathrm{~S}$ Calcd for $(\mathrm{M}+\mathrm{H})^{+}: 313.0754$. Found: 313.0762.

$\mathbf{N}$-(4-Fluorophenyl)-5-(pyridin-3-yl)-1,3,4-thiadiazole-2-carboxamide (180). Colorless crystals (69\%); m.p. 200-

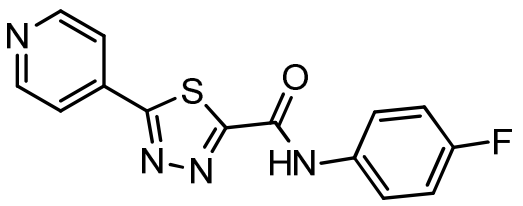

$202{ }^{\circ} \mathrm{C} .{ }^{1} \mathrm{H}$ NMR $\left(300 \mathrm{MHz}, \mathrm{DMSO}-d_{6}\right) \delta 11.35$ (s, 1H, NH), 9.27 (s, 1H, Py), 8.81 $(\mathrm{d}, J=4.0 \mathrm{~Hz}, 1 \mathrm{H}, \mathrm{Py}), 8.49$ (d, J=7.9 Hz, 1H, Py), 7.92-7.8 (m, 2H, Ar), $7.72-$ $7.58(\mathrm{~m}, 1 \mathrm{H}, \mathrm{Py}), 7.31-7.25(\mathrm{~m}, 2 \mathrm{H}, \mathrm{Ar}) .{ }^{13} \mathrm{C}$ NMR (75 MHz, DMSO-d $) \delta 169.6$ (C, thiadiazole), 166.6 (C=0), 160.9 (C, thiadiazole), 156.4 (1C, Py), 153.0 (1C, Py), 148.9 (1C, Py), 135.9 (1C, Py), 124.9 (1C, Py), 123.3 (2C, Ar), 123.2 (1C, 
Ar), 116.0 (2C, Ar), 115.7 (1C, Ar). HRMS (ESI) C ${ }_{14} \mathrm{H}_{9} \mathrm{FN}_{4} \mathrm{OS}$. Calcd for $(\mathrm{M}+\mathrm{H})^{+}:$301.0554. Found: 301.0550.

N-(2,5-Dimethoxyphenyl)-5-(pyridin-3-yl)-1,3,4-thiadiazole-2-carboxamide (18p). Yellow crystals (40\%); m.p.

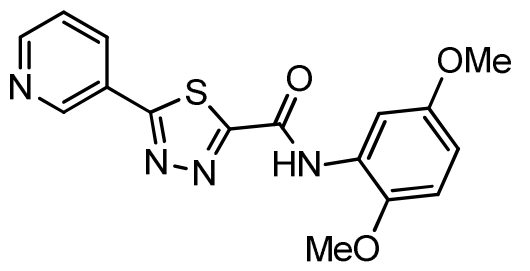
199-200 ${ }^{\circ} \mathrm{C} .{ }^{1} \mathrm{H}$ NMR (300 MHz, CDCl $) \delta 9.83(\mathrm{~s}, 1 \mathrm{H}, \mathrm{NH}), 9.27$ (s, 1H, Py), 8.82 $(\mathrm{d}, J=4.7 \mathrm{~Hz}, 1 \mathrm{H}, \mathrm{Py}), 8.38(\mathrm{~d}, J=8.0 \mathrm{~Hz}, 1 \mathrm{H}, \mathrm{Ar}), 8.19(\mathrm{~d}, J=2.7 \mathrm{~Hz}, 1 \mathrm{H}, \mathrm{Py})$, 7.60-7.48 (m, 1H, Py), $6.90(\mathrm{~d}, J=8.9 \mathrm{~Hz}, 1 \mathrm{H}, \mathrm{Ar}), 6.71(\mathrm{~d}, J=9.0 \mathrm{~Hz}, 1 \mathrm{H}, \mathrm{Ar})$, $3.95\left(\mathrm{~s}, 3 \mathrm{H}, \mathrm{CH}_{3}\right), 3.85\left(\mathrm{~s}, 3 \mathrm{H}, \mathrm{CH}_{3}\right) .{ }^{13} \mathrm{C} \mathrm{NMR}\left(75 \mathrm{MHz}, \mathrm{CDCl}_{3}\right) \delta 169.4(\mathrm{C}$, thiadiazole), 165.69 (C, thiadiazole), 155.4 (1C, C=0), 153.5 (1C, Ar), 152.3 (1C, Py), 148.8 (1C, Py), 142.7 (1C, Ar), 135.2 (1C, Py), 128.5 (1C, Py), 126.1 (1C, Py), 128.0 (1C, Ar), 112.4 (1C, Ar), 110.5 (1C, Ar), 104.3 (1C, Ar), 56.0 (1C, CH $\mathrm{CH}_{3}$, 55.7 (1C, CH ( $_{3}$. HRMS (ESI) $\mathrm{C}_{16} \mathrm{H}_{14} \mathrm{~N}_{4} \mathrm{O}_{3} \mathrm{~S}$. Calcd for $(\mathrm{M}+\mathrm{H})^{+}:$343.0859. Found: 343.0852 .

$\mathbf{N}$-(2,5-Dimethoxyphenyl)-5-(pyridin-4-yl)-1,3,4-thiadiazole-2-carboxamide (18q). Orange crystals (40\%); m.p.<smiles>COc1ccc(OC)c(NC(=O)c2nnc(-c3ccncc3)s2)c1</smiles>
220-222 ${ }^{\circ} \mathrm{C} .{ }^{1} \mathrm{H}$ NMR (300 MHz, $\left.\mathrm{CDCl}_{3}\right) \delta 9.63(\mathrm{~s}, 1 \mathrm{H}, \mathrm{NH}), 8.85$ (d, J=4.7 Hz, $2 \mathrm{H}, \mathrm{Py}), 8.37$ (d, J=8.9 Hz, 1H, Ar), 7.93 (d, J = 4.5 Hz, 2H, Py), 6.60-6.52 (m, $2 \mathrm{H}, \mathrm{Ar}), 3.96\left(\mathrm{~s}, 3 \mathrm{H}, \mathrm{CH}_{3}\right), 3.86\left(\mathrm{~s}, 3 \mathrm{H}, \mathrm{CH}_{3}\right) \cdot{ }^{13} \mathrm{C} \mathrm{NMR}\left(75 \mathrm{MHz}, \mathrm{DMSO}-d_{6}\right)$ low solubility. HRMS (ESI) $\mathrm{C}_{16} \mathrm{H}_{14} \mathrm{~N}_{4} \mathrm{O}_{3} \mathrm{~S}$. Calcd for $(\mathrm{M}+\mathrm{H})^{+}$: 343.0859. Found: 343.0854 .

N-(2,6-Dimethoxyphenyl)-5-(pyridin-4-yl)-1,3,4-thiadiazole-2-carboxamide (18r). Yellow crystals (40\%); m.p.<smiles>COc1cccc(OC)c1NC(=O)c1nnc(-c2ccncc2)s1</smiles>
190-192 ${ }^{\circ} \mathrm{C} .{ }^{1} \mathrm{H}$ NMR $\left(300 \mathrm{MHz}, \mathrm{CDCl}_{3}\right) \delta 8.85$ (d, J = $\left.5.8 \mathrm{~Hz}, 2 \mathrm{H}, \mathrm{Py}\right), 8.59(\mathrm{~s}, 1 \mathrm{H}$, $\mathrm{NH}), 7.91(\mathrm{~d}, J=5.8 \mathrm{~Hz}, 2 \mathrm{H}, \mathrm{Py}), 7.32(\mathrm{~m}, 1 \mathrm{H}, \mathrm{Ar}), 6.69(\mathrm{~s}, 1 \mathrm{H}, \mathrm{Ar}), 6.66(\mathrm{~s}, 1 \mathrm{H}, \mathrm{Ar})$, $3.89\left(\mathrm{~s}, 6 \mathrm{H}, \mathrm{CH}_{3}\right) .{ }^{13} \mathrm{C} \mathrm{NMR}\left(75 \mathrm{MHz}, \mathrm{CDCl}_{3}\right) \delta 170.0$ (C, thiadiazole), 166.8 (C, thiadiazole), 160.7 (C=O), 152.1 (2C, Py), 148.9 (1C, Py), 150.0 (2C, Ar), 125.9 (2C, Py), 113.6 (1C, Ar), 111.0 (2C, Ar), 104.1 (1C, Ar), 57.5 (2C, CH $)$. HRMS (ESI) $\mathrm{C}_{16} \mathrm{H}_{14} \mathrm{~N}_{4} \mathrm{O}_{3} \mathrm{~S}$. Calcd for $(\mathrm{M}+\mathrm{H})^{+}: 343.0859$. Found: 343.0850 .

\section{Acknowledgements}

This work was supported by Russian Science Foundation (RSF grant 14-50-00126)

\section{References}

1. Haider, S.; Alam, M. S.; Hamid, H. Eur J. Med. Chem. 2015, 92, 156-177. https://dx.doi.org/10.1016/i.ejmech.2014.12.035

2. Srivastava, K.; Purohit, S.; Singhal, S. Asian J. Biomed. Pharm. Sci. 2013, 3, 6-23.

3. Ananikov, V. P.; Khokhlova, E. A.; Egorov, M. P.; Sakharov, A. M.; Zlotin, S. G.; Kucherov, A. V.; Kustov, L. M.; Gening, M. L.; Nifantiev N. E. Mendeleev Commun. 2015, 25, 75-82. https://doi.org/10.1016/j.mencom.2015.03.001

4. Joseph, L.; George, M.; Mathews, P. J Pharm Chem Biol Sci. 2015, 3, 329-345.

5. Bretschneider, T.; Franken, E. M.; Görgens, U.; Fusslein, M.; Hense, A.; Kluth, J. 2011, US Patent 0098287.

6. Gintsburg, A. L.; Zigangirova, N. A.; Zayakin, E. S.; Luyksaar, S. I.; Kapotina, L. N. 2012, RU Patent 2495036 (in Russian).

7. Nardi, A.; Ratcliffe, P.; Craan, T.; Hertrampf, T.; Lesch, B.; Kime, R.; Steinhagen, H. 2015, WO Patent 161928. 
8. Singh, S. J.; Rajamanickam, S.; Gogoi, A.; Patel, B. K. Tetrahedron Lett. 2016, 57, 1044-1047. https://dx.doi.org/10.1016/j.tetlet.2016.01.083

9. Krayushkin, M. M.; Yarovenko, V. N.; Zavarzin, I. V. Rus. Chem. Bull. 2004, 53, 517-527. https://dx.doi.org/10.1023/B:RUCB.0000035631.82058.9c

10. Yarovenko, V. N.; Krayushkin, M. M.; Zayakin, E. S.; Zorina, V. V.; Kapotina, L. N.; Zigangirova, N. A. J. Chem. Eng. 2010, 4, 55-59.

11. Krayushkin, M. M.; Yarovenko, V. N.; Zayakin, E. S.; Gintsburg, A. L.; Zigangirova, N. A.; Zorina, V. V.; Tokarskaya, E. A.; Tartakovskaya, D. I.; 2010, RU Patent 2400471 (in Russian).

12. Yarovenko, V. N., Shirokov, A. V., Zavarzin, I. V., Krupinova, O. N., Ignatenko, A. V., Krayushkin, M. M. Chem. Heterocycl. Comp. 2003, 39, 1633-1639.

https://doi.org/10.1023/B:COHC.0000018342.57499.16

13. Aroyan, A. A. Synth. Heterocycl. Comp., Yerevan 1972, 9, 8 (in Russian).

14. Yarovenko, V. N.; Shirokov, A. V.; Krupinova, O. N.; Zavarzin, I. V.; Krayushkin, M. M.; Russ. J. Org. Chem.; 2003, 39(8), 1133-1139.

http://dx.doi.org/10.1023/B:RUJO.0000010181.01921.77 